DOI: 10.17707/AgricultForest.62.1.28

GRYAZ'KIN A.V., GUROV S.V., BELIAEVA N.V., KOVALEV N.V., SOKOLOVA V.A. ${ }^{1}$

\title{
A MODEL OF NATURAL RENEWAL OF PINE TREES IN TAIGA ZONE
}

\begin{abstract}
SUMMARY
The dynamic of the natural pine trees renewal process in Taiga zone based on mathematical modelling is examined. Dynamic modelling is used for prediction of natural renewal success depending on seeds' yield and bumper crop years' recurrence. In current study mean bumper crop recurrence is assumed to be once per 5 years, this means that a bumper crop year may occur in 4 years as well as in 6 years. The analysis of the state of natural renewal depending on maternal stand among main forest types is made. The bearing age of 30 years is assumed, with the maximum seed productivity age of 110-130 years, depending on the forest type. The data on trunk quantity distribution depending on age was obtained as a result of a simple sum. The algorithm of the sum has been implemented in Interpol procedure contained in the initial text file gav4.cpp. The initial distribution of trunk quantity depending on age was taken from the growth progress tables. The change of trunk quantity by decades was determined using extrapolation of known values according to power function $\mathrm{N}=424316,4 \times \mathrm{A}-$ 1,3533 . The value of approximation certainty factor equals $\mathrm{R}^{2}=0.9857$. The age of stands changes from 20 to 190 years. The amount of bearing trees aged 31-40 years old was assumed as 5-10\%, whereas at the maximum bearing age (110-130 years old) the share of bearing trees totals $70-90 \%$ depending on the forest type. The presented model is discrete, dynamic, stochastic and descriptive. Stochasticity of the process is caused by the uncertainty in the quantity of bearing trees depending on age, uncertainty in the quantity of seeds from a single tree, uncertainty in the quantity of sprouts and in the occurrence of normal and bumper crops. To account for randomness a function ravnom $(a, b)$ has been introduced, which generates uniformly distributed random numbers from a to $b$. Implementation of this function is possible using $\mathrm{C}++$ language. Due to the stochasticity of the natural renewal process more implementations of the program are needed for a more reliable prediction.
\end{abstract}

Keywords: natural renewal, pine tree, logging, tree stand, live groundcover.

\section{INTRODUCTION}

Dynamic models are an innovative tool for evaluating possible changes in the structure and state of forest phytocenoses [1, 2, 3, 4, 5]. Mathematical

\footnotetext{
${ }^{1}$ Gryaz'kin A.V., Gurov S.V., Beliaeva N.V.,Kovalev N.V., Sokolova V.A, Saint-Petersburg State Forest Technical University under the name of S.M. Kirov, Saint-Petersburg, RUSSIA.

Notes: The authors declare that they have no conflicts of interest. Authorship Form signed online.
} 
modeling is widely used in Russia's forestry complex [9, 10]. Dynamic modeling can also be used for predicting success of natural renewal depending on main factors which define the process' success: forest environment, maternal stand age, seed yield, recurrence of bumper crop years. Due to that it is necessary to draw attention of native researchers to modeling of natural processes. The first step towards that is development of existing models by means of their calibration and validation for use in certain forest environments. The goal of this study is the development of NAFOR model as the means of a long-term prediction of forest ecosystems renewal potential in north-western Russia.

\section{Pine tree seed productivity}

Seed bearing years-once per 5-6 years;

Bearing start age- 30 years;

Quantity of bearing trees aged 31-40 years old is 5-10\%; at the maximum bearing age (130-160 years old) the share of bearing trees must be 70$90 \%$ (in following years the share of bearing trees is decreased each decade, down to $5-10 \%$ at $300-400$ years old age);

number of seeds from a single tree is 400 in the first 10 years;

each following decade 100 seeds are added (10 each year, or differentially: 1-2 in the first year, 2-4 in the second year, 3-6 in the third year etc.) up till the age of 110 in an oxalis type forest, of 120 in a bilberry type, of 130 in a long moss type;

the peak of bearing is in the age of 110 in the oxalis type forest, 120 in the bilberry type, 130in the long moss type;

further, the quantity of seeds is reduced by 100 in each following decade until the end of the life (by 10 each year, or differentially: 1-2 in the first year, 2-4 in the second year, 3-6 in the third year etc.);

each year 1 to $3 \%$ of seeds become sprouts; each year 50 to $95 \%$ of sprouts die off;

1 to $10 \%$ of trees live up to bearing age.

\section{Prediction of trunk quantity (by age stages)}

The pine starts bearing at the age of 30 . The prediction must be made for 200 years. Therefore, $A m i n=30, A m a x=200$. The initial distribution of trunk quantity depending on age is obtained from tables of growth progress [8]. Change of trunk quantity by decades was determined by extrapolating known values according to power function $\mathrm{N}=424316,4 \times \mathrm{x}-1,3533$. Age variable A varies from 20 to 190 years old. Approximation certainty factor equals R2= 0,9857 which is the evidence of the model's adequacy. The approximating function's appearance and its parameters have been obtained by programmatic means. Graphical results of approximation are presented on Figure 1. Curve 1 corresponds with initial data about trunk quantity by age classes, whereas curve 2 corresponds to modeling results. 


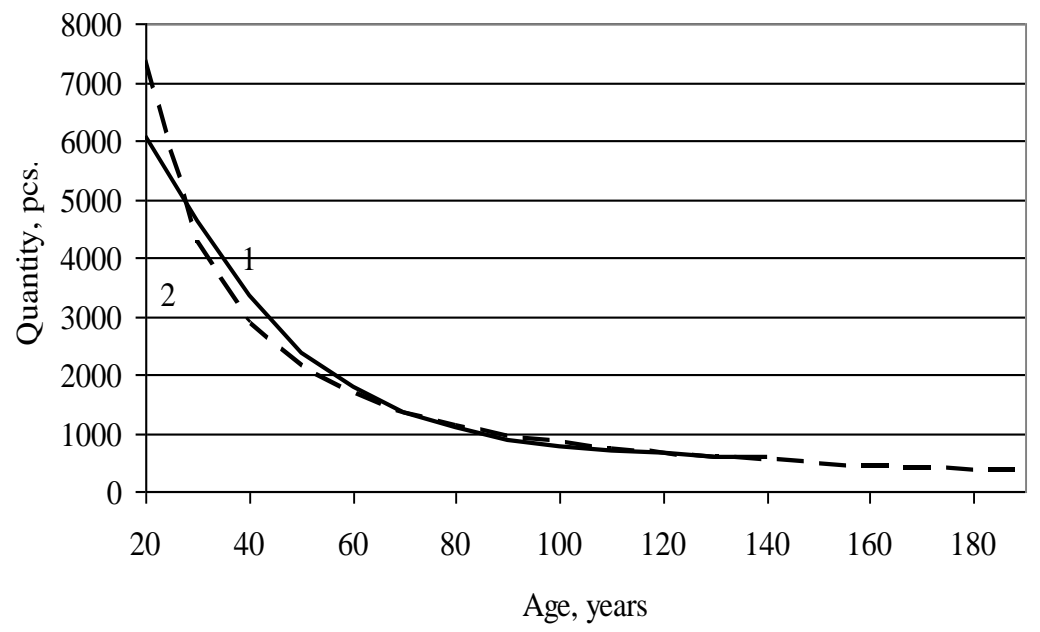

Figure 1. Approximation results (quantity of trunks)

The second stage which is tied to conversion of initial data is the decrease of the tree age interval to one year. For this purpose we will study the age change interval from $A$ to $A+10$ years, where $A$ is divisible by 10 . Let the trunk quantity of $A$ age equal $N(A)$ and the trunk quantity of $A+10$ age equal $N(A+10)$. For each $A+i, i=0,1,2 \ldots, 9$ age we will define new trunk quantity of $n(A+i)$ trees of $A+i$ age in order for it to be linear according to $i$, to form a decreasing sequence and for the equation $\sum_{i=0}^{9} n(A+i) N(A)$ to be true. The algorithm of this sum's solution has been implemented in Interpol procedure which is contained in the initial text file gav4.cpp. As a result, we will obtain data on the trunk quantity distribution according to their age. It is worth noting that counting is performed from the bearing start age equaling $A_{\min }=30$ years old till $A_{\max }=200$ years old. The next step is adding seed quantity, sprout quantity and quantity of underbrush starting from 1 year old age to $A_{\min }$. This is achieved by performing approximation using a power function $N=92948,7 \times A^{-1,5064}$. The approximation certainty is $R^{2}=0,9884$.

\section{Forest renewal process description}

The idea of modelling forest renewal process is in an opportunity to obtain information about a pine tree's renewal potential during $t$ time point if there is information available from the previous time point $t-1$. The information is as follows:

-age and quantity of bearing trees;

-quantity of seeds appearing in the initial year;

-quantity of sprouts appearing in the next year;

-quantity of underbrush appearing in the next 3-5 years;

-quantity of trees reaching bearing age. 
Above information depends on the whole previous history of the forest renewal process $[6,7,9]$. With that said, if any part of the information is absent, valuable prediction of further forest evolution is practically impossible. Thus, from the mathematical point of view, we are dealing with a multivariate random process.

To predict the process' development it is necessary to account for all factors which influence the process.

Complete information about the state of the forest tract in respect to the quantity of trunks is as follows:

-quantity of trunks $N(a)$ with age $A$ if $A \geq A_{\min }$;

-quantity of seeds (sprouts, underbrush) with age $A$ if $A<A_{\text {min }}$.

If this information is known at the time point $t-1$, it is sufficient for obtaining similar information at $t$ time point. Then, it is possible to trace the changes in the data from the starting year till any subsequent one. Thus, the concept model of the natural forest renewal is quite simple. The other matter is formalizing the model, i.e. obtaining recurrent correlations which allow to mathematically following the process' evolution.

According to classification of all kinds of mathematical models, current model is discrete, dynamic, stochastic and descriptive. Stochasticity of the process is caused by the uncertainty in the quantity of bearing trees depending on age, uncertainty in the quantity of seeds from a single tree, uncertainty in the quantity of sprouts and in the occurrence of normal and bumper crops.

Let the massive $A u[t]$ characterize normal and bumper crop years. If $A u[t]=0$ - the year $t$ is normal, if $A u[t]=1$ - the year $t$ is a bumper crop year. For a pine tree bumper crop years occur, approximately, once per 5 years. This means that the bumper crop year may occur in 4 years as well as in 6 years.

To account for randomness a function ravnom $(a, b)$ has been introduced, which generates uniformly distributed random numbers from $a$ to $b$. Implementation of this function is possible using $\mathrm{C}++$ language.

\section{RESULTS AND DISCUSSION}

Determining quantity of bearing trees

Based on the initial data we receive a random implementation of the bearing trees percentage depending on their age. The time interval from $A_{\min }$ to $A_{\max }$ can be divided into 3 or 5 stages depending on bearing intensity. Let us examine the case where bearing intensity changes within 5 stages with the increase of the stand age. At the first, the third and the fifth stages the quantity of bearing trees is roughly constant, at the second stage it increases and at the fourth stage it decreases. Thus for a pine tree the first stage is defined by the age of trees from $A_{n 1}=31$ years old to $A_{n 2}=40$ years old. Within this interval the quantity of bearing trees is from $p_{n 1}=5 \%$ to $p_{n 2}=10 \%$. The third stage is defined by the maximum bearing age of trees from $A_{s l}=130$ years old to $A_{s 2}=160$ years old. The percentage of bearing trees within this interval is from $p_{s l}=70 \%$ to $p_{s 2}=90 \%$. The fifths stage corresponds to the age from $A_{k l}=300$ years old to $A_{k 2}=400$ years old. 
The percentage of bearing trees within this interval is from $p_{k l}=5 \%$ to $p_{k 2}=10 \%$.

By using linear extrapolation we will determine the percentage of bearing trees within the second and the fourth intervals.

The second interval corresponds to the age of trees from $A_{n 2}=40$ years old to $A_{s l}=130$ years old. The range of percentage variation at the beginning of the second stage must be from $p_{n 1}$ to $p_{n 2}$ and the range of percentage variation at the end of the second interval must be from $p_{s 1}$ to $p_{s 2}$.

The fourth interval corresponds to the age of trees from $A_{s 2}=160$ years old to $A_{k l}=300$ years old. Thus, the range of percentage variation at the beginning of the fourth stage must be from $p_{s 1}$ to $p_{s 2}$ and the range of percentage variation at the end of the fourth interval must be from $p_{k 1}$ to $p_{k 2}$.

If the percentage of bearing trees may vary from $p_{1}$ to $p_{2}$, then for a normal year we will use the function ravnom $\left(p_{1}, p_{2}\right)$,and for a bumper crop year we will consider that the percentage of bearing trees is maximum and equals $p_{2}$. Mathematically this can be presented as follows. Let $A_{n 1} \leq A \leq A_{n 2}$ (first interval), then the percentage of bearing trees can be expressed as a following ratio:

\section{$P(A)=\left\{\begin{array}{c}\text { ravnom }\left(p_{n 11}, p_{n 2}\right) \text {, if the year is a normal one } \\ p_{n \mathbf{2}} \text {, if the year is a bumper crop one }\end{array}\right.$.}

Let $A_{n 2}<A<A_{s l}$ (second interval), then the percentage of bearing trees can be can be expressed as a following ratio:

\section{$P(A)=\left\{\begin{array}{c}\text { ravnom }\left(p_{1}, p_{\mathbf{2}}\right) \text {, if the year is a normal one } \\ p_{\mathbf{2}}, \text { if the year is a bumper crop one }\end{array}\right.$,}

where

$$
p_{1}=p_{n 1}+\boldsymbol{\zeta}_{s 1}-p_{n 1} \ni \frac{A-A_{n 2}}{A_{s 1}-A_{n 2}}, p_{2}=p_{n 2}+\boldsymbol{\bigotimes}_{s 2}-p_{n 2} \ni \frac{A-A_{n 2}}{A_{s 1}-A_{n 2}} \text {. }
$$

Let $A_{s 1} \leq A \leq A_{s 2}$ (third interval), then the percentage of bearing trees can be expressed as a following ratio:

$$
P(A)=\left\{\begin{array}{c}
\text { ravnom }\left(p_{s 1}, p_{s 2}\right) \text { if the year is a normal one } \\
p_{s 2}, \text { if the year is a bumper crop one }
\end{array} .\right.
$$

Let $A_{s 2}<A<A_{k l}$ (fourth interval), then the percentage of bearing trees can be can be expressed as a following ratio:

\section{$P(A)=\left\{\begin{array}{c}\text { ravnom }\left(p_{1}, p_{\mathbf{2}}\right) \text {, if the year is a normal one } \\ p_{\mathbf{2}} \text {, if the year is a bumper crop one }\end{array}\right.$,}

where

$$
p_{1}=p_{s 1}+\boldsymbol{\bigotimes}_{k 1}-p_{s 1} \ni \frac{A-A_{s 2}}{A_{k 1}-A_{s 2}}, p_{2}=p_{s 2}+\boldsymbol{\bigotimes}_{k 2}-p_{s 2} \ni \frac{A-A_{s 2}}{A_{k 1}-A_{s 2}} \text {. }
$$

Let $A_{k 1} \leq A \leq A_{k 2}$ (fifth interval), then the percentage of bearing trees can be expressed as a following ratio: 


\section{$P(A)=\left\{\begin{array}{c}\text { ravnom }\left(p_{k 1}, p_{k 2}\right) \text {, if the year is a normal one } \\ p_{k 2} \text { if the year is a bumper crop one }\end{array}\right.$}

The value of bearing trees percentage during a normal and a bumper crop year depending on age is easily calculated. At that, the age varies from 31 to 200 years old with an interval of one year.

Graphical illustration of $P(A)$ function is given on Figure 2.

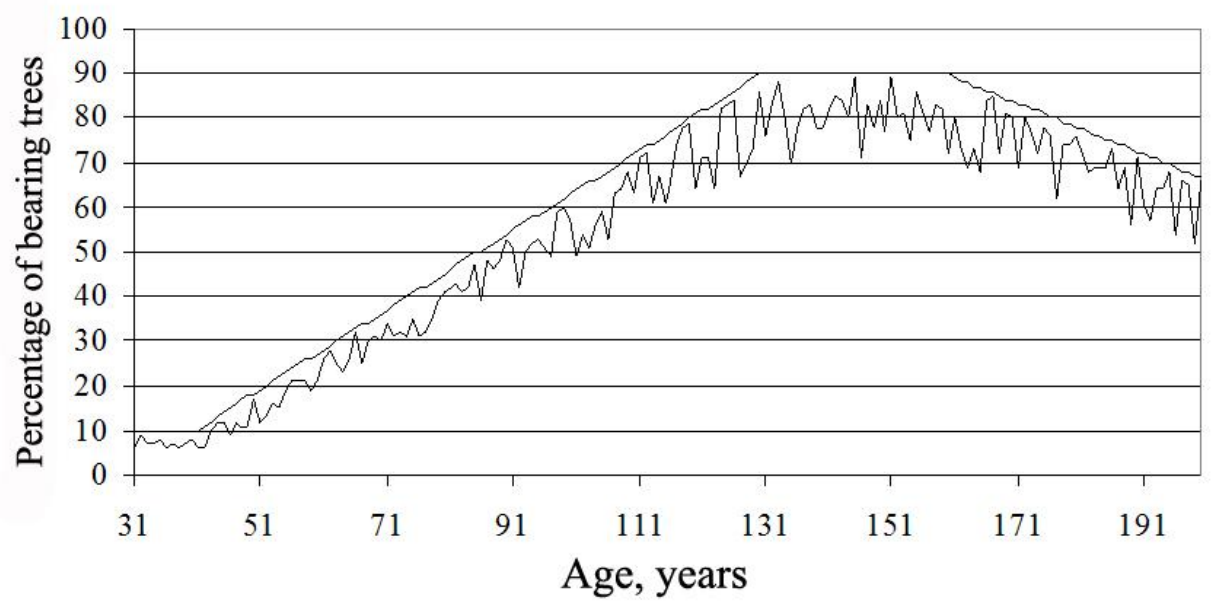

Figure 2.Variation of bearing trees quantity with the change of age

Serrated curve on Figure 2 is one of bearing trees percentage implementations for a normal year. Upon further executions of the program this implementation will be different. A number of such implementations gives an idea about variation of bearing trees percentage depending on their age. The second line which changes smoothly defines the bearing trees percentage for a bumper crop year.

Determining seeds quantity from a single tree

Let us examine another important characteristic - a forest's seed productivity. Its variation depending on age also corresponds to a certain implementation of the random process equaling a number of seeds from a single tree of $A$ age. According to initial data the time interval from $A_{\min }$ to $A_{\max }$ can also be presented in 5 stages. At the first, the third and the fifth stage the quantity of seeds from a tree is roughly constant, at the second stage this quantity increases and at the fourth stage it decreases. For a pine tree the first stage is defined by the age of trees from $A_{n 1}=31$ years old to $A_{n 2}=40$ years old. During this stage the quantity of seeds from a single tree is $m_{n 1}=m_{n 2}=400$. The third stage is defined by the maximum bearing age of trees from $A_{s l}=110$ years old to $A_{s 2}=130$ years old depending on a forest type. During this stage the quantity of seeds from a single tree reaches $m_{s 1}=m_{s 2}=1100$. The fifths stage corresponds to the age from $A_{k l}=300$ years old to $A_{k 2}=400$ years old. The quantity of seeds at this point is so small that it can be considered zero: $m_{k l}=m_{k 2}=0$. 
Same as in the previous item, to determine the number of seeds at the second and the fourth stages we will use linear extrapolation.

The second stage corresponds to the age of trees from $A_{n 2}=40$ years old to $A_{s l}=110$ years old. The range of a single tree seed quantity variation at the beginning of the second stage must equal $m_{n l}=m_{n 2}=400$, and the range of a single tree seed quantity variation at the end of the second stage must equal $m_{s 1}=m_{s 2}=1100$.

The second stage corresponds to the age of trees from $A_{s 2}=130$ years old to $A_{k l}=300$ years old. Due to that the range of seed quantity variation at the beginning of the second stage must equal $m_{s 1}=m_{s 2}=1100$ and the range of seed quantity variation at the end of the second stage must equal zero: $m_{k 1}=m_{k 2}=0$.

It is necessary to account for a fact that within these intervals the quantity of seeds is a random value with defined mean values. If the seed quantity varies from $\mathrm{m} 1$ to $\mathrm{m} 2$, then for a normal year we will use the function ravnom $\left(m_{1}, m_{2}\right)$ and for a bumper crop year we will consider that the seed quantity from a single tree is maximum and equals $m_{2}$. Then we will obtain the following ratios for determining seed quantity from a single tree.

Let $A_{n 1} \leq A \leq A_{n 2}$ (first interval), then the seed quantity from a single tree is expressed as a following ratio:

$$
M(A)=\left\{\begin{array}{cc}
\text { rawnom }\left(m_{n 1}, m_{n 2}\right) \text { if the year is a normal one } \\
m_{n 2}, \quad \text { if the year is a bumper crop one }
\end{array}\right.
$$

Let $A_{n 2}<A<A_{s l}$ (second interval), then the number of seeds is expressed as a ratio:

$$
(A)=\left\{\begin{array}{cc}
\operatorname{rawnom}\left(m_{1}, m_{2}\right) \text { if the year is a normal one } \\
m_{2}, \quad \text { if the year is a bumper crop one }
\end{array}\right.
$$

where

$$
\begin{array}{r}
m_{1}=m_{n 1}+\left(n_{s 1}-m_{n 1} \doteq \frac{A-A_{n 2}}{A_{s 1}-A_{n 2}},\right. \\
m_{2}=m_{n 2}+\left(n_{s 2}-m_{n 2} \doteq \frac{A-A_{n 2}}{A_{s 1}-A_{n 2}} .\right.
\end{array}
$$

Let $A_{s 1} \leq A \leq A_{s 2}$ (third interval), then the number of seeds is expressed as a ratio:

$$
M(A)=\left\{\begin{array}{cc}
\operatorname{rawnom}\left(m_{s 1}, m_{s 2}\right) \text { if the year is a normal one } \\
m_{s z_{2}} \text { if the year is a bumper crop one }
\end{array}\right.
$$
ratio:

Let $A_{s 2}<A<A_{k 1}$ (fourth interval), then the number of seeds is expressed as a

$$
\text { (A) }=\left\{\begin{array}{c}
\operatorname{ravnom}\left(m_{1}, m_{2}\right) \text { if the year is a normal one } \\
m_{2}, \quad \text { if the year is a bumper crop one }
\end{array}\right.
$$

where

$$
m_{1}=m_{s 1}+n_{k 1}-m_{s 1} \doteq \frac{A-A_{s 2}}{A_{k 1}-A_{s 2}},
$$




$$
m_{2}=m_{s 2}+\boldsymbol{C}_{k 2}-m_{s 2} \doteq \frac{A-A_{s 2}}{A_{k 1}-A_{s 2}} .
$$
ratio:

Let $A_{k 1} \leq A \leq A_{k 2}$ (fifth interval), then the number of seeds is expressed as a

$$
M(A)=\left\{\begin{array}{cc}
\text { ravnom }\left(m_{k \mathbf{1}}, m_{k \mathbf{z}}\right) \text {, if the year is a normal one } \\
m_{k \mathbf{2}}, \quad \text { if the year is a bumper crop one }
\end{array}\right.
$$

Graphical illustration of $M(A)$ function is given on Figure 3.

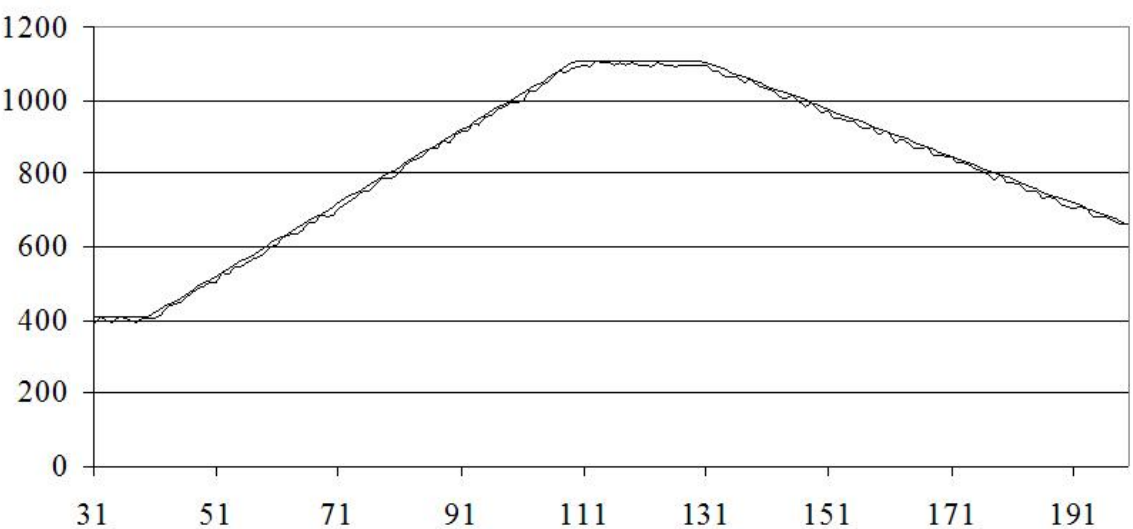

Age, years

Figure 3. Variation of seed quantity from a single tree with the change of its age

A smooth upper line on Figure 3 reflects the change of seed quantity from a single tree for a bumper crop year. The lower changing line represents an implementation of the seed quantity from a single tree for a normal year. This implementation changes randomly but within preset limits. A combination of such implementations gives an idea about the change of seed quantity depending on the age of trees.

For a more precise forming of these lines it is necessary to account for the forest type and for primary forest estimation characteristics of studied stands. However, this condition doesn't considerably influence prediction of tree quantity.

Determining sprout quantity

The percentage of surviving sprouts and underbrush decreases with age $A$ and towards the bearing age their remainder makes up from $V_{n} \%$ to $V_{k} \%$ relative to initial seed quantity. By modifying these values it is possible to receive different predictions of trunk quantity. Let $V_{n}=40 \%$ and $V_{k}=55 \%$. Then linear extrapolation will give us the percentage of surviving sprouts depending on their age: 


$$
V(A)=\left\{\begin{array}{c}
100-\left(100-\operatorname{ravnom}\left(V_{n}, V_{k}\right)\right) \times \frac{A-1}{A_{\min }-1} \text {, if the year is a normal one } \\
100-\left(100-V_{k}\right) \times \frac{A-1}{A_{\min }-1}, \text { if the year is a bumper crop one }
\end{array}\right.
$$

The age changes from 1 year to $A_{\min }=30$ years (for a pine tree).

\section{CONCLUSIONS}

Study of the natural forest renewal is a complex task, no matter what the type of a tree it is. Dynamic modelling was used to predict successfulness of natural renewal depending on maternal stand age, seed yield and bumper crop years' recurrence accounting for the type of the forest. Modelling of the process is tied to the necessity of performing multiple iterations of the developed program for obtaining various initial data. Such necessity is caused by the uncertainty in the bearing trees' quantity at each age stage, in the seed quantity from a single tree, in the share of sprouts from the total seed quantity, in a combination of normal and bumper harvests of seeds at different stages of stand development. Due to stochasticity of the process a large number of program's implementations are needed.

\section{ACKNOWLEDGEMENTS}

This study was supported by the Scientific Research Projects Office of the Saint-Petersburg State Forest Technical University under the name of S.M. Kirov, Saint-Petersburg, Russia.

\section{REFERENCES}

Beliaeva N.V., Gryaz'kin A.V. Main aspectsof Taiga zonefur-woods` renewal. LesnoyeHozyaystvo (Forestry). 2011. № 4. P. 21-23.

Beliaeva N.V., Danilov D.A. Patterns of natural renewal at forest maintenance and complex forest maintenance felling objects. Izvestiya SanktPeterburgskoylesotehnicheskoyakademii (St.Petersburg Forest Technical Academy news). 2009. Issue 188. P. 30-39.

Gryaz'kin A.V. Taiga forests' renewal potential. St.Petersburg: SPbGLTA, 2001. 188 p.

Gryaz'kin A.V. Structureand potential of under brushunderuneven-agedfur-woodcanopy. M.: VINITI, 1994. 51 p.

Gryaz'kin A.V., Smirnov A.P. Influence of economical actions on structural elements of forest biogeocenoses. M.: VINITI, 1997. $74 \mathrm{p}$.

Gryaz'kin A.V. Structural organization of phytocenosesin Southern Taiga (by the example of fur-woods of the green moss forest type group). St.Petersburg: SPbGLTA, 1999. 136 p.

Sokolov A.I. Forest renewal at felling sites of North-Western Russia. Petrozavodsk: Karelia NZ RAN (Scientific center of Russian Academy of Sciences). 2006. 215 p.

Tetyukhin S.V., Minayev V.N., Bogomolova L.P. Forest estimation and regulation: normative and reference sources concerning the North-West of the Russian Federation.St.Petersburg: SPbGLTA, 2005. 360 p. 
Fetisova A.A. Multiple classification of Pinussilvestris L.natural renewal success in Leningrad region conditions: Candidate of Biological Studies Dissertation: 06.03.02. St.Petersburg: SPbGLTA, 2013. 162 p.

Chertov O.G., Gryaz'kin A.V., Komarov A.S., Smirnov A.P., Beliaeva N.V., Vavilov S.V., Paramonov S.G., Smirnov A.A., Sokolova V.A., Kovalev N.V. etal. Dynamic modeling in forestry. St.Petersburg: SPbGLTA, 2011. 66 p. 